\title{
Krisenbearbeitung durch digitale Plattformen
}

Rezension zu Moritz Altenried / Julia Dück / Mira Wallis (Hg.) (2021):

Plattformkapitalismus und die Krise der sozialen Reproduktion.

Münster: Westfälisches Dampfboot.

Barbara Orth

Abb. 1 Titel des Buches (Quelle: Westfälisches Dampfboot)

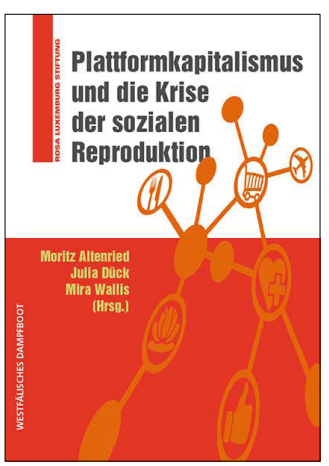

Digitale Plattformen und die wissenschaftliche Auseinandersetzung mit ihnen haben - auch bedingt durch die Corona-Lockdowns - einen Aufschwung erfahren. Viele der von Plattformen angebotenen Dienstleistungen erweisen sich in der Krise als wichtige Infrastrukturen. Für die Aufrechterhaltung des Arbeitsalltags haben sich Kommunikationsplattformen wie zum Beispiel Zoom als essenziell erwiesen, während Ausgangssperren und die Schließung vieler Geschäfte dazu führten, dass Konsumgüter des alltäglichen Lebens wie zum Beispiel Haushaltsgegenstände und Kleidung vermehrt über OnlinePlattformen wie Amazon bestellt wurden. Nicht zuletzt haben digitale Dienstleistungsplattformen wie Essens- und Lebensmittellieferdienste dafür gesorgt, dass Menschen auch in Quarantäne weiterhin mit dem Notwendigsten versorgt blieben.

Der im Verlag Westfälisches Dampfboot erschienene Sammelband Plattformkapitalismus und die Krise der sozialen Reproduktion, herausgegeben von Moritz Altenried, Julia Dück und Mira Wallis, setzt hier an. In 14 Beiträgen wird der Aufstieg digitaler Plattformen nicht als reine technologische Weiterentwicklung diskutiert, sondern vielmehr in direkten Zusammenhang mit langfristigen gesellschaftlichen Konjunkturen gesetzt. Zentral für den Sammelband ist die Annahme, dass die neoliberale Wirtschaftspolitik der letzten Jahrzehnte zu einer Verknappung der Zeit für nicht bezahlte Sorgearbeit führt. Diese Krise sozialer Reproduktion fungiert als Folie, vor der aufgezeigt wird, warum es überhaupt zur rasanten Ausbreitung der sogenannten Gig-Economy[1] kam. Neben drei Übersetzungen aus dem Englischen bilden die Beiträge das aktuelle Forschungsfeld Gig-Economy in den deutschsprachigen Sozialwissenschaften umfassend und interdisziplinär ab. So bietet der Band auch eine Bestandsaufnahme des noch neuen Forschungsfeldes Plattform-Urbanismus.

Schon im ersten Teil des Buches wird der Forschungsgegenstand multiperspektivisch und kritisch betrachtet: Wie lassen sich digitale Plattformen in die kontinuierliche Entwicklung kapitalistischer Akkumulationsregime einordnen? Wie lässt sich eine Krise der Reproduktion nicht nur deskriptiv, sondern auch analytisch fassen? Und: Was zeichnet digitale Plattformen 
eigentlich aus? Gerade letztere Frage zu Definitionen und Konzeptualisierungen von Plattformen wird unter Plattform-Forschenden durchaus kontrovers diskutiert. Während einige Forschende digitale Plattformen als gewinnmaximierende Firmen beschreiben, deren Geschäftsmodell auf dem Sammeln von Daten beruht (vgl. van Doorn/Adam 2020), konzeptualisieren andere Forschende Plattformen als Infrastrukturen, die Daten, Personen und Dienstleistungen effizient koordinieren (vgl. Richardson 2020). Der zweite Teil des Sammelbandes widmet sich der Verräumlichung und Digitalisierung von Reproduktionsarbeiten. Auf diese theoretischen Einordnungen folgen im dritten und vierten Teil empirische Beiträge. Diese geben qualitative Einblicke in den Alltag digital vermittelter Dienstleistungen aus der Perspektive von GigArbeiter_innen. Im fünften und letzten Teil des Bandes werden die Möglichkeiten und Grenzen genossenschaftlich organisierter Plattformen diskutiert.

Die Beiträge des Bandes verdeutlichen, dass Plattformen zwar durch Logiken des Investmentkapitals entstanden und geprägt sind, aber gleichzeitig auch einen Modus der Krisenbearbeitung darstellen. Denn kapitalistische Produktionsweisen führen einerseits zu einer Krise der sozialen Reproduktion, indem sie eine Überausbeutung des Individuums und eine Entgrenzung des (Lohn-)Arbeitstages forcieren. Andererseits bringen kapitalistische Produktionsweisen technologische Innovationen hervor, in diesem Fall in Form der Dienstleistungsplattformen, die Individuen wiederum eine Lösung für ihren Zeitnotstand anbieten, nämlich ihre Reproduktionsarbeit an prekär beschäftigte Gig-Arbeiter_innen auszulagern. Auf diesen roten Faden verweisen die Herausgeber_innen in ihrer Einleitung, indem sie Plattformarbeit nicht als einzigartiges Phänomen, sondern „als ein Labor flexibilisierter und digitalisierter Arbeit" (Altenried/Dück/Wallis 2021b: 14) verstehen. Im Folgenden gehe ich auf einzelne Beiträge ein, die mir entweder aufgrund ihrer theoretischen Ansätze als zentral erscheinen oder besonders spannende empirische Befunde vorstellen.

Die ersten beiden Kapitel umreißen die beiden Forschungsfelder des Sammelbandes. Julia Dück vermittelt einen Einstieg in die Diskussionen zur Krise der Reproduktion und Moritz Altenried skizziert die Grundzüge und Besonderheiten des Plattformkapitalismus. Es folgt eine Übersetzung von Ursula Huws' Beitrag zu Digitalisierung und Hausarbeit, der die Krise sozialer Reproduktion mit dem Aufkommen digitaler Plattformen in Verbindung setzt und somit einen hilfreichen theoretischen Rahmen für die empirischen Beiträge des Sammelbandes bietet. Huws analysiert den zunehmend expansiven und intensiven Charakter moderner Arbeitsverhältnisse, die regelmäßige Überstunden und ständige Erreichbarkeit durch digitale Technologien erfordern. Mit einer überzeugenden Typologisierung verschiedener Arten von Reproduktionsarbeit gelingt es Huws nachzuzeichnen, dass Individuen immer weniger Zeit für substanzielle Reproduktionsarbeit übrig bleibt. Damit arbeitet sie die doppelte Verstrickung digitaler Plattformen in kapitalistische Akkumulationsregime heraus: Zum einen sind Plattformen selbst ein Produkt kapitalistischer Krisen - sie setzten sich nach der Finanzkrise 2007/o8 durch. Zum anderen schaffen kapitalistische Produktionsweisen genau den eklatanten Zeitnotstand in privaten Haushalten, der einen Markt für bezahlte Dienstleistungen im Haushalt hervorbringt. Dieser wiederum kann nun von Plattformunternehmen bedient werden. 
Auch Nick Srnicek und Helen Hester sind bereits prägend für die Plattform-Forschung. Die Übersetzung ihres Textes zur Geschichte der Technologisierung des Haushalts leitet den zweiten Teil des Buches ein. Der Beitrag besticht vor allem durch eine Vielzahl amüsanter Anekdoten und vermittelt die historische Entwicklung von Haushaltsgeräten mit einer wunderbaren Leichtigkeit. Der_Die Leser_in erfährt, dass die Erfindung der Mikrowelle im Jahre 1950 die letzte technische Innovation war, die eine nennenswerte Zeitersparnis in der Hausarbeit einbrachte. Seit mehr als siebzig Jahren also lässt die (feministische) Hoffnung, Maschinen könnten lästige Hausarbeit irgendwann gänzlich übernehmen, auf sich warten. Im Gegenteil: Die Autor_innen merken an, dass das Smarthome seinen Bewohner_innen verglichen mit herkömmlichen Geräten sogar zusätzliche Zeit abverlange. Der Beitrag relativiert somit indirekt die unter Technologieunternehmen weitverbreitete Annahme, dass der Notstand in Pflege und Sorgearbeit durch den Einsatz von mehr Technologie gelöst werden könne (vgl. Prestia 2019).

Von der Mikroperspektive einzelner Haushalte springen Yannik Ecker, Marcella Rowek und Anke Strüver im folgenden Beitrag auf die städtische Ebene. Sie führen in die Debatten um Plattform-Urbanismus ein, die im Umfeld kritischer Stadtgeographie sowie feministischer und digitaler Geographie in den letzten Jahren entstanden sind (z. B. Leszczynski 2020; Richardson 2020; Sadowski 2020; vgl. auch Altenried/Animento/ Bojadžijev 2021, in s u b $\backslash$ u r b an 9/1-2). Die Autor_innen legen dabei einen Schwerpunkt auf neue Arbeitsbereiche wie bezahlte Pflege- und Sorgearbeit, die durch Plattformen erschlossen werden.

Im dritten Teil des Sammelbandes werden diese theoretischen Überlegungen um empirische Erkenntnisse ergänzt. Lisa Bors auto-ethnographischer Zugang als Putzkraft, die über die Vermittlungsplattform Helpling tätig wird, zeigt zum Beispiel, dass Helpling nicht vorrangig die obere Mittelschicht bedient. Gegenüber der etablierten Literatur zu Hausarbeit, die die Beschäftigung von Hausangestellten als Distinktionsmerkmal eines wohlhabenden Milieus eingeordnet hatte (vgl. Anderson 2000), scheint das Gig-Modell den breiten Durchschnitt der Gesellschaft als Kund_innen anzusprechen. Hier ergänzen die Beiträge des Sammelbandes Erkenntnisse aus ähnlichen aktuellen Forschungsprojekten in der Schweiz (Keller/ Schwiter 2021) und bestätigen Eindrücke aus explorativen quantitativen Erhebungen (Huws et al. 2017).

Während reproduktive Tätigkeiten wie Kochen, Putzen, Pflege und Einkaufen zunehmend gigifiziert und somit auch zu Erwerbsarbeit werden, bleibt unbezahlte Reproduktionsarbeit jedoch weiterhin eine Voraussetzung für jedwede Erwerbsarbeit inklusive der Gig-Arbeit. Diesen Zusammenhang arbeitet Simiran Lalvani in ihrem Beitrag heraus. Anhand von Interviews mit Gig-Arbeiter_innen in Mumbai zeigt Lalvani, dass deren Überausbeutung auf der Auslagerung ihrer eigenen Reproduktionsarbeit basiert. Mit anderen Worten: Nur wer zu Hause bekocht wird, kann lange Schichten als prekäre_r Gig-Arbeiter_in überhaupt stemmen. In dem einzigen außereuropäischen Beitrag des Sammelbandes überzeugt Lalvani auch durch die detaillierte Analyse des Gig-Modells im südasiatischen Kontext. Hinduistische Vorstellungen von Reinheit markieren dort bestimmte Situationen, aber auch ganze Gruppen als spirituell unrein. So gelten menstruierende 
Lieferdienst-Arbeiter_innen ebenso als unrein wie Angehörige bestimmter Kasten, woraus sich andere Fragen und Probleme für Essenslieferdienste ergeben, als bisher in der Plattform-Literatur beschrieben. Somit gelingt es Lalvani, die viel beforschten Essenslieferdienste aus einer neuen, intersektionalen Perspektive zu betrachten. Während Plattform-Forschung lange Zeit vorwiegend die ökonomische Prekaritätvon Gig-Arbeit betonte, geht die Autorin darauf ein, wie diese entlang von Differenzlinien unterschiedlich stark ausgeprägt ist. Zudem erforscht sie, wie sich die Erfahrungen der GigArbeiter_innen durch verschiedene Positionierungen entlang von Gender, Kaste, Klasse und religiöser Markierungen unterscheiden, anstatt ihre Subjektivierungsprozesse als implizit männlichen Durchschnitt zu setzen (vgl. Orth 2021).

Auf die empirischen Beiträge folgen Vicky Kluziks theoretische Überlegungen zur Subjektivierung der Gig-Arbeiter_innen als feminisierte, rassifizierte Individuen. Auch dieser Beitrag eröffnet neue Perspektiven für eine differenztheoretische Betrachtung des Plattform-Urbanismus.

Der vierte Teil des Sammelbandes wendet sich wieder der empirischen Forschung zu, allerdings im Feld von Gig-Arbeit, die vollständig im digitalen Raum stattfindet, der sogenannten Crowdwork. Im Gegensatz zu digital vermittelten, aber analog ausgeführten Tätigkeiten ist Crowdwork - eine entsprechende Internetverbindung vorausgesetzt - vollkommen ortsunabhängig. Mira Wallis konstatiert in ihrem Beitrag, dass sich Unternehmen mit Crowdwork „lokal unterschiedliche Reproduktionsbedingungen” (Wallis 2021: 248) zunutze machen können. Ihre vergleichende Forschung in Rumänien und Deutschland verdeutlicht, dass die multiplication of labour (Mezzadra/Neilson 2013) kein neues Phänomen ist, aber durch den Einsatz von Technologien noch effizienter ausgebeutet werden kann. Wie Mohammed Anwar und Mark Graham (2018) ausführten, habe der Plattformkapitalismus somit das Problem einer ortsgebundenen Arbeiter_innenschaft in diesem Sektor gelöst. Weiterhin analysiert Wallis, dass Crowdwork nicht nur eine räumliche Überlappung von Produktions- und Reproduktionsort bedeutet. Vielmehr finde auch eine zeitliche Fragmentierung sowie eine Kommodifizierung des privaten Umfeldes statt. Künstliche Intelligenzen wie Amazons Alexa müssen mit den Daten möglichst unterschiedlicher Nutzer_innenprofile gespeist werden, damit sie auch ältere Menschen oder Menschen mit verschiedenen Dialekten und Akzenten verstehen können. So werden Crowdworker_innen etwa dazu angehalten, ihre Familienangehörigen zum Einsprechen verschiedener Beispielsätze zu bewegen oder ihre Wohnumfelder zu fotografieren. Smarthome-Algorithmen lernen so am Haushalt des_der Crowdworker_in.

Darauf folgend skizzieren Wiebke Frieß und Iris Nowak erste Erkenntnisse aus ihrer noch laufenden Erhebung zu den Erfahrungen von Crowdworker_innen mit Behinderung. Sie beschreiben, dass sich Arbeiter_innen mit Behinderungen zwar durch Assistenztechnologien wie Bildschirmlesehilfen im digitalen Raum teils barriereärmer bewegen können als an einem analogen Arbeitsplatz. Gleichzeitig stellen die Autor_innen aber fest, dass Crowdwork durch die hohe Flexibilität, die Kleinteiligkeit und die ständige Verfügbarkeit, die den Arbeiter_innen abverlangt wird, besonders herausfordernd ist. Somit scheint Crowdwork ebenso wenig eine technologische Lösung für 
Barrieren im physischen Raum zu sein, wie es das Smarthome für die Zeitnot ist. Beide empirischen Beiträge zu Crowdwork illustrieren außerdem, dass Gig-Arbeitsverhältnisse vor allem dann als außergewöhnlich prekär einzuordnen sind, wenn sie mit dem sogenannten Normalarbeitsverhältnis verglichen werden. Letzteres gab und gibt es in vielen Branchen und Ländern bzw. für bestimmte Gruppen schlicht nicht. So bezahlen Werkstätten für Menschen mit Behinderung durchschnittlich 1,30-1,50 Euro pro Stunde (Wenzel-Warkentin 2021) und auch für viele Migrant_innen, Frauen, ältere Menschen oder Menschen ohne Aufenthaltsstatus erscheint Gig-Arbeit nicht außergewöhnlich prekär zu sein.

Im abschließenden Teil greift Jonas Pentzien eine in der Literatur bereits vorgebrachte Forderung auf, Plattformen als Kooperativen zu organisieren (z. B. Anwar/Graham 2018; Scholz 2017). Sein Beitrag stellt einen vorsichtig optimistischen Ausblick auf die Zukunft des Städtischen dar.

Insgesamt bietet der Sammelband einen sehr umfassenden, kritischen und gut kuratierten Überblick zum Forschungsfeld Plattformen und Reproduktion. Da sich die meisten Forschungsbeiträge zur Plattform-Ökonomie bisher vor allem auf Fahrdienste, Wohnungsvermietung und Essensauslieferung konzentrieren, setzen die Herausgeber_innen mit der Auswahl der Beiträge einen wichtigen Impuls, um bestehende Forschungslücken zu schließen.

Da über Fahr- und Lieferdienstplattformen überdurchschnittlich viele männliche Arbeiter tätig sind und diese statistisch gesehen weniger oft Reproduktionsarbeit neben ihrer Lohnarbeit leisten, ist aus bisheriger Forschung kaum etwas darüber bekannt, ob und wie Gig-Modelle Geschlechterverhältnisse zu Hause verändern. Huws geht sogar so weit, von einem „Versagen der wachsenden Forschungslandschaft zu digitaler Arbeit, die sich kaum mit den Auswirkungen von Online-Plattformen auf die geschlechtsspezifische Arbeitsteilung innerhalb und außerhalb des Haushalts auseinandersetzt“ (Huws 2021: 88), zu sprechen. Hier markiert der Sammelband eine Trendwende, denn viele der Beiträge setzen sich ganz explizit mit der vergeschlechtlichten Ungleichverteilung von Haus- und Sorgearbeit auseinander.

Weiterhin basieren die bisher breit beforschten Plattformen überwiegend auf Geodaten, während die weniger beforschten Sorge-Plattformen fast ausschließlich über Sortierungslogiken funktionieren (Ticona/ Mateescu 2018). Dies bedeutet, dass ein Algorithmus Nutzer_innen nicht aufgrund ihres räumlichen Abstandes zueinander verbindet, sondern lediglich eine Vorsortierung verschiedener Anbieter_innenprofile übernimmt. Anhand dieses Rankings kann der_die Kund_in dann selbst eine_n passende_n Anbieter_in auswählen. Diese Unterschiede sind nicht nur technisch relevant, sondern verschaffen auch intersektionalen Forschungsdesigns eine besondere Relevanz: Unter anderem können sie sichtbar machen, welche Strategien Arbeitende verfolgen, um die repressive Logik von Plattformen zu unterlaufen. Statt Plattformen als übermächtige Vertreter des Kapitals darzustellen und in eine „techno-hysteria” (Leszczynski 2020: 190) zu verfallen, können intersektionale Analysen, wie sie in Plattformkapitalismus und die Krise der sozialen Reproduktion versammelt sind, zu einem differenzierteren Verständnis des Forschungsfeldes führen. Gleichzeitig weist der wiederholte Rückgriff auf 
Srniceks (2016) Plattform-Typologisierung[2] aber auch darauf hin, dass die theoretische Auseinandersetzung mit dem Plattform-Urbanismus noch am Anfang steht und weiterer Bearbeitung bedarf. Der Sammelband ist also allen zu empfehlen, die sich für Digitalisierung, Urbanismus, feministische Theorie und Gig-Economy interessieren und sich intensiver mit diesem Forschungsfeld auseinandersetzen wollen.

\section{Dieser Artikel wurde durch den Publikationsfonds der Freien Universität Berlin gefördert.}

\section{Endnoten}

[1] Als Gig-Economy wird derjenige Sektor des Plattformkapitalismus bezeichnet, in dem Plattformen digital mediierte Dienstleistungen anbieten und diese pro Stück/Gig bezahlen. Gig-Arbeitskräfte sind nicht fest angestellt, sondern arbeiten freiberuflich auf eigenes Risiko.

[2] Diese Typologisierung umfasst fünf verschiedene Arten digitaler Plattformen: Plattformen, deren Geschäftsmodell auf Werbung basiert (z. B. Google, Facebook), Cloud-Plattformen, die vorwiegend Speicherkapazitäten stellen (z. B. Amazon Web Services, Salesforce), Industrieplattformen (z. B. Siemens), Produktplattformen (z. B. Spotify) und sogenannte schlanke Plattformen, zu denen klassische Gig-Plattformen wie Uber oder Airbnb zählen (vgl. Srnicek 2016).

\section{Autor_innen}

Barbara Orth interessiert sich für feministische Theorie, (post)migrantische Ansätze und urbane Bewegungen. Aktuell forscht sie zu digitalen Plattformen.

barbara.orth@fu-berlin.de

\section{Literatur}

Altenried, Moritz / Animento, Stefania / Bojadžijev, Manuela (2021): Plattform-Urbanismus: Arbeit, Migration und die Transformation des urbanen Raums. In: sub\urban. zeitschrift für kritische stadtforschung 9/1-2, 73-91.

Altenried, Moritz / Dück, Julia / Wallis, Mira (2021a): Plattformkapitalismus und die Krise der sozialen Reproduktion. Münster: Westfälisches Dampfboot.

Altenried, Moritz / Dück, Julia / Wallis, Mira (2021b): Zum Zusammenhang digitaler Plattformen und der Krise der sozialen Reproduktion: Einleitung. In: Moritz Altenried / Julia Dück / Mira Wallis (Hg.), Plattformkapitalismus und die Krise der sozialen Reproduktion. Münster: Westfälisches Dampfboot, 7-28.

Anderson, Bridget (2000): Doing the dirty work? The global politics of domestic labour. London/New York: Zed Books.

Anwar, Mohammed / Graham, Mark (2018): Labour. In: James Ash / Rob Kitchin / Agnieszka Leszczynski (Hg.), Digital geographies. Thousand Oaks: Sage, 177-185.

van Doorn, Niels / Badger, Adam (2020): Platform capitalism's hidden abode. Producing data assets in the gig economy. In: Antipode 52/5, 1475-1495.

Huws, Ursula (2021): Der Ärger mit dem Haushalt: Digitalisierung und Kommodifizierung von Hausarbeit. In: MoritzAltenried/Julia Dück/Mira Wallis(Hg.), Plattformkapitalismus und die Krise der sozialen Reproduktion. Münster: Westfälisches Dampfboot, 70-92.

Huws, Ursula / Spencer, Neil / Syrdal, Dag / Holts, Kaire (2017): Work in the European gig economy. Research results from the UK, Sweden, Germany, Austria, the Netherlands, Switzerland and Italy. Brussels: Foundation for European Progressive Studies, 1-58. 
Keller, Marisol / Schwiter, Karin (2021): Unsichtbar in der Gig Economy: Feministische Perspektiven und autoethnographische Methoden zur Erforschung der Gigifizierung von Care-Arbeit. In: Feministische Geo-RundMail 85, 13-16. https://ak-feministischegeographien.org/rundmail/ (letzter Zugriff am 30.9.2021).

Leszczynski, Agnieszka (2020): Glitchy vignettes of platform urbanism. In: Environment and Planning D: Society and Space 38/2, 189-208.

Mezzadra, Sandro / Neilson, Brett (2013): Border as method, or, the multiplication of labor. Durham: Duke University Press.

Orth, Barbara (2021): Engaging feminist geographies in platform research. In: Feministische Geo-RundMail 85, 16-22. https://ak-feministische-geographien.org/rundmail/ (letzter Zugriff am 30.9.2021).

Prestia, Angela S. (2019): Leveraging the gig economy. A novel solution to improve health care costs. In: Nurse Leader 17/4, 356-359.

Richardson, Lizzie (2020): Coordinating the city. Platforms as flexible spatial arrangements. In: Urban Geography 41/3, 458-461.

Sadowski, Jathan (2020): Cyberspace and cityscapes. On the emergence of platform urbanism. In: Urban Geography 41/3, 448-452.

Scholz, Trebor (2017): Uberworked and underpaid. How workers are disrupting the digital economy. Cambridge/Malden: Polity Press.

Srnicek, Nick (2016): Platform capitalism. Cambridge/Malden: Polity Press.

Ticona, Julia / Mateescu, Alexandra (2018): Trusted strangers. Carework platforms' cultural entrepreneurship in the on-demand economy. In: New Media \& Society 20/11, 4384-4404.

Wallis, Mira (2021): Digitale Arbeit und soziale Reproduktion: Crowdwork in Deutschland und Rumänien. In: Moritz Altenried / Julia Dück / Mira Wallis(Hg.), Plattformkapitalismus und die Krise der sozialen Reproduktion. Münster: Westfälisches Dampfboot, 228-251.

Wenzel-Warkentin, Natalia (2021): Lohn in Behindertenwerkstätten: „Euer Taschengeld brauch’ ich nicht“. In: Frankfurter Allgemeine Zeitung, 1.5.2021. https://www.faz.net/ aktuell/gesellschaft/menschen/stundenlohn-von-rund-1-50-euro-was-man-in-einerbehindertenwerkstatt-verdient-17308441.html (letzter Zugriff am 15.6.2021). 